\title{
Supervision und Soziale Arbeit - historisch verwandt, praktisch verbunden, konzeptuell entfernt
}

\author{
Stefan Busse
}

Eingegangen: 5. Februar 2021 / Angenommen: 16. Februar 2021 / Online publiziert: 6. April 2021

(C) Der/die Autor(en) 2021

Zusammenfassung Die Supervision ist schon lange keine (reine) Sozialarbeitssupervision mehr. Sie wird heute als ein generalisiertes berufs- und arbeitsweltorientiertes Beratungsformat verstanden. Dennoch sind Supervisor/innen heute beratend hauptsächlich in Feldern Sozialer Arbeit tätig. Gleichzeitig ist ihre Geschichte im deutschsprachigen Raum als eine konzeptuelle Wegbewegung von der Sozialen Arbeit zu beschreiben. Diese ist als eine Gegenstands-, Zuständigkeits- und Kompetenzerweiterung rekonstruierbar. Ihre zentralen Professionalisierungsanforderungen bestehen jedoch im Umgang mit zentralen Widersprüchen der Spätmoderne, was sie wiederum mit ihrem „Ursprungsland“ der Sozialen Arbeit teilt.

Schlüsselwörter Supervision · Soziale Arbeit · Coaching · Organisationsberatung

Dieser Beitrag ist eine stark überarbeitete Fassung meines Buchbeitrags: A German Perspective on Supervision: Supervision between maintenance of, emancipation from or abnegation of the origins of social work? (Busse 2021, in press).

Prof. Dr. S. Busse $(\bowtie)$

Hochschule Mittweida, Technikumplatz 17, 09648 Mittweida, Deutschland

E-Mail: busse@hs-mittweida.de 


\title{
Supervision and social work-historically related, practically connected, conceptually removed
}

\begin{abstract}
The supervision has long ceased to be a (pure) social work supervision. Today it is understood as a generalized professional and work-oriented consulting format. Nevertheless, today supervisors are mainly active in the areas of social work. At the same time, their history in German-speaking countries can be described as a conceptual move away from social work. This can be reconstructed as an extension of the subject, responsibility and competence. Its central professionalization requirement, however, consist in dealing with central contradictions of late modernism, which it in turn shares with its "country of origin" of social work.
\end{abstract}

Keywords Supervision $\cdot$ Social work $\cdot$ Coaching $\cdot$ Organizational consulting

\section{Soziale Arbeit und Supervision - ein irritierendes Bild}

Schaut man auf die Beziehung zwischen Supervision und Sozialer Arbeit, ergibt sich ein irritierendes Bild. Die Supervision ist schon lange keine (reine) Sozialarbeitssupervision mehr. Sie versteht sich heute als ein generalisiertes berufs- und arbeitsweltorientiertes Beratungsformat und muss diesen Anspruch mit anderen Beratungsformaten (vor allem mit der Organisationsberatung und dem Coaching) teilen.

Dennoch, wenn man schaut, wo Supervisor/innen ${ }^{1}$ heute hauptsächlich beratend tätig sind, dann stehen wir vor einer etwas paradoxen Situation: Erstens ist die Supervision historisch eng mit der Geschichte der Sozialen Arbeit verbunden. Zweitens ist die Soziale Arbeit heute noch immer das wichtigste Beratungsfeld von Supervisor/ innen. Die aktuelle Mitgliederbefragung der DGSv (2020) bestätigt die gewohnte Situation. Fast die Hälfte der bereits 2018 befragten Supervisoren und Coaches boten zu Dreiviertel und mehr ihre Beratungsleistung in „sozial orientierten Feldern“ an (Brutzer de Palma 2019, S. 31). Gegenwärtig sind es sogar fast 83\%, die dezidiert in der Sozialen Arbeit beratend unterwegs sind, gefolgt von fast $77 \%$, die im Feld von „Kindheit, Jugend und Familie“ als klassischem Feld Sozialer Arbeit Beratung (Supervision und Coaching) anbieten. Alle anderen Berufsfelder (im Nonfor-Profit-, im produktiven Bereich und im Dienstleistungsbereich) außerhalb der Sozial- und der Gesundheitswirtschaft werden deutlich unter $10 \%$ angegeben.

Drittens: Die Supervision hat sich konzeptuell weit von der Sozialen Arbeit entfernt, ihre widersprüchliche Professionalisierungsgeschichte lässt sich aber nur als lang bestehende Ko-Evolution mit der Sozialen Arbeit verstehen (Busse 2009, 2021). Und sie bleiben über Grundfragen sozialer resp. professionalisierter Dienstleistung in der spätmodernen Lebens- und Arbeitswelt eng verbunden. Zugleich sollte man nicht ignorieren, dass all dies z.T. auch auf Sonderentwicklungen in der deutschsprachigen (z. T. auch europäischen) Supervisionscommunity verweist. So ist z. B. in den US-amerikanischen, australischen, neuseeländischen und südostasiatischen

\footnotetext{
1 Im Folgenden wird nicht durchweg die gendergerechte Sprachform gewählt; selbstverständlich sind mit „Supervisoren“, „Berater“, „Klienten“ usw. immer alle Geschlechter gemeint.
} 
Supervisionsdiskursen die Soziale Arbeit nicht nur ein Beratungsfeld, sondern weiterhin ein wichtiger Referenzraum, in dem Supervision konzeptuell weiterentwickelt wird (Yuen-han Mo et al. 2020; Engelbrecht und O'Donoghue 2021).

\section{Entwicklungslinien und Widersprüche der Professionalisierungsgeschichte der Supervision}

\subsection{Supervision im Dienste der Professionalisierung Sozialer Arbeit}

„Die Geschichte der Supervision ist eigentlich zunächst die Geschichte der Sozialarbeit" (Wieringa 1990, S. 34). Damit ist für die amerikanische Entwicklung des Verhältnisses von Supervision und Sozialer Arbeit, aber auch für die deutsche nach dem Zweiten Weltkrieg Wesentliches gesagt (ausführlich Belardi 1992). Ihre Entwicklungspaten waren in den 1950er und 1960er-Jahren vor allem re-emigrierte Psychoanalytiker, Sozialarbeiter und Ausbilder von Sozialarbeitern aus den USA resp. England (exemplarisch Louis Lowy; vgl. Stenzel 2019), die im Rahmen von Re-Edukationsprogrammen der westdeutschen Gesellschaft nach dem Zweiten Weltkrieg agierten. So gesehen hatte Supervision eine frühe Bindung an das Demokratisierungsprojekt in der BRD.

Auch niederländische Sozialarbeiter/innen und Supervisor/innen (z. B. Cora Baltussen; vgl. Austermann 2019; oder Cornelis F. Wieringa und Henk Foole) boten an den deutschen Wohlfahrtsschulen (später in den höheren Fachschulen) im Rahmen des Casework (der Einzelfallhilfe), aber auch der Gruppenarbeit (Group Work) Supervision als Einübung in das methodische Handeln und als Reflexion der Helfer-Klienten-Interaktion an. Supervision wurde so zu einer besonderen Lehr- und Vermittlungsform sozialarbeiterischen Handelns. Sie war eine Art ,caseworking the caseworker" (Huppertz 1975, S. 57). Ein reflexives Verständnis von Supervision hat sich aber erst allmählich herausgebildet - durch eine Differenzierung von Praxisanleitung, Praxisberatung und Praxisreflexion (Hege 2011, S. 60).

Das konzeptuelle Spektrum, was unter Supervision verstanden wurde, bewegte sich zu dieser Zeit zwischen Pragmatismus eines ,,ausgeprägten sozialarbeiterischen Selbstverständnisses“, konzeptuellen Orientierungen an Gruppendynamik und Psychoanalyse und ersten Sensibilisierungen für den organisationalen und gesellschaftlichen Kontext von Sozialer Arbeit (und Supervision) (Leuschner und Weigand 2011, S. 42). Die frühen Fortbildungsinstitutionen für Soziale Arbeit und Supervision haben so auch die erste Generation von deutschen Supervisoren hervorgebracht, die wiederum als Pioniere die weitere Institutionalisierung der Supervision vorangetrieben haben. Sie waren dann auch die Protagonisten eines schwierigen Prozesses, eine verbandliche „Plattform für fachlichen Austausch und eine gemeinsame Interessenvertretung“ zu finden, der schließlich zur Verbandsgründung der DGSv führte (Lippenmeier 2011, S. 12). Bis heute besteht eine große Verbundenheit mit dieser ersten Generation und ein Bedürfnis, sich der frühen auch demokratischen Wurzeln der Supervision zu vergewissern (vgl. Lohl 2019). 


\subsection{Die kritisch emanzipatorische Prägung der Supervision}

Die Soziale Arbeit als „Ursprungsland“ der Supervision hat sich im Rahmen der Reformbewegungen der 1960er-Jahre selbst verändert und eine i.W. bis heute gültige wohlfahrtsstaatliche Fassung bekommen. Es ist ein Wohlfahrtssystem entstanden, in dem sich die „Sozialwirtschaft" zwischen Staat und Markt als ein dritter Sektor herausgebildet hat. Soziale Arbeit agiert hier bekanntlich in einem „sozialhilferechtlichen Dreiecksverhältnis“ - zwischen staatlichen „Leistungsträgern“ (z.B. einem Jugendamt), einem „Leistungserbringer“(den sogenannten ,,freien Trägern“) und schließlich den Hilfebedürftigen als „Leistungsberechtigen“ (vgl. von Schubert 1999; Pattar 2012). Soziale Arbeit (bzw. die gesamte Sorgearbeit) wird bis heute im Spannungsfeld dieser triadischen Struktur erbracht. Von hier aus ergeben sich dann auch einige Reflexionsbedarfe für Supervision, die über das Einüben methodischen Handelns (Praxisanleitung), aber auch über das bloße Reflektieren dieses Einübens (Praxisberatung) hinausgehen. Mit der Kritik an der sozialarbeiterischen Einzelfallhilfe als ,individualistischer Introspektion auf das Seelische“ (Hege 1979, S. 46) wird die Forderung verbunden, die Einzelfallhilfe als einen ,engagierten Dialog “ und ihr Pendant die Fallsupervision ausgehend von einem ,emanzipatorische(n) Fallverständnis“ zu entwickeln (Althoff 2018, S. 11). Die Supervision galt nämlich einigen (nicht allen) Vertretern der Sozialen Arbeit selbst als ein ,problematisches Kapitel der Sozialarbeit“, die als „Zauberformel amerikanischer Provenienz“ eher eine sedierende Wirkung habe und Sozialarbeiter in einer marginalen Situation halte (vgl. Huppertz 1975, S. 149).

Die Frage, die von hier ausging, ist bis heute virulent: Kann oder soll Soziale Arbeit resp. Supervision im Widerspruch zwischen Emanzipation und Anpassung „Sand oder Schmiermittel im Getriebe sein“ (Leuschner und Weigand 2011)? Die Ablösung vom Ursprungsland amerikanischer Prägung führte zu einer An-Bindung an das Verständnis einer kritischen Professionalität, als die sich die bundesdeutsche Soziale Arbeit zu verstehen begann. Insofern sollte die Supervision eher zu einer kritischen Begleiterin der professionellen Selbstkontrolle Sozialer Arbeit werden (so bereits Huppertz 1975, S. 157). Damit war ein kritisch-emanzipatorischer Anspruch formuliert, der mit einer bürokratisch konservierenden Praxis heftig kollidierte (Blinkert und Huppertz 1974). Dieses Selbstverständnis hat bis heute weite Teile der Community der Supervisoren kritisch-emanzipatorisch imprägniert (Gröning 2016); es umfasst die Frage nach einem eigenen professionellen Mandat für die Supervision. Daran ändert auch der unlängst von Lohl erbrachte Befund nichts, dass man heute mit historischem Abstand einräumen muss, dass dies kein durchgängig geteiltes Verständnis dieser ersten Pioniergeneration gewesen ist (vgl. Lohl 2019, S. 44-51).

\subsection{Die „Verberuflichung“ der Supervision}

Neben und nach dieser emanzipatorischen Prägung der Supervision, die sie mit der Sozialen Arbeit noch geteilt hat, schien sie sich selbst von der Sozialen Arbeit als alleinigem Beratungsfeld ,emanzipieren“ bzw. ihre Zuständigkeiten erweitern zu wollen. Wenn beide auch heute noch ,ziemlich beste Freundinnen“ zu sein scheinen, 
ist Supervision in andere Berufsgruppen und -felder expandiert. Die Supervision hat sich ,,verberuflicht“".

Die Idee, „dass Supervision auch für andere Berufe verwendet ..., auf andere Berufsgruppen (Supervisanden S.B.) angepasst werden könne", gehe bereits auf Louis Lowy (1983) zurück (Stenzel 2019, S. 26). Zum einen drängten auch andere Berufsgruppen in den Supervisionsmarkt (Psychotherapeuten, Pädagogen, Theologen, Psychologen, Soziologen etc.) und machten den Sozialarbeitern ,ihr“ neues prestigeträchtiges Berufsfeld streitig. Von daher mussten sogar Widerstände in der Community der Supervisoren überwunden werden, Nicht-Sozialarbeiter (vor allem Psychologen) in den Verband der DGSv aufzunehmen (Weigand 2012, S. 20). ${ }^{2}$ Die DGSv war kein (zweiter) Sozialarbeiter-Verband mehr.

Zum anderen vollzog sich als Gegenbewegung dazu die Expansion (,vertikale Expansion“; Kühl 2006) der Supervision in Tätigkeitsfelder außerhalb der Sozialen Arbeit. Eine generalisierte Berufs- und Arbeitsfeldorientierung (Gotthardt-Lorenz und Walther 1998) führte schließlich an die kritische Grenze, an der nicht nur das Feld der Sozialen Arbeit, sondern auch das Feld von Professionals und damit der Non-For-Profitbereich überschritten wurden. Diese Öffnung zum For-Profit-Bereich wurde als „Faszination des Geldes und des fremden Feldes“ (Weigand 1993) durchaus ambivalent und ,als steiniger Weg in andere Arbeitsfelder“ (Fürstenau 1995) wahrgenommen. Hier wurde die Supervision nicht nur mit einer anderen Berufslogik, sondern mit der Logik eines anderen und konträren gesellschaftlichen Funktionssystems konfrontiert. So ging es um die bange oder hoffnungsfrohe Frage (je nach Standpunkt des Supervisoren), ob Supervision auch hier gut zu platzieren sei (vgl. Diebäcker 1995; Buchinger 1999). Konfrontiert mit dem eigenen emanzipatorischen Selbstanspruch war die Leitfrage dieser Entwicklung nicht allein die nach dem Verhältnis von Beratungs- und Feldkompetenz, sondern auch, wo die Trennlinie zwischen „selbstreflexiver“ und ,instrumenteller bzw. strategischer“ Beratung verläuft (Leuschner und Diebäcker 1995).

Diese ursprünglich vielleicht nur pragmatisch motivierte Absatzbewegung der Supervision von ihrem Ursprungsland zeitigt bis heute identitätspolitische Nachwehen. Wenn Supervision schon nicht mehr Sozialarbeitssupervision ist (und sein kann), so solle sie doch Beratung für professionelle Berufe oder doch für Berufstätigkeiten aller Art sein, sofern sie nur irgendwie einen gewissen Reflexionsbedarf aufweisen? Hier stehen sich bis heute ein engeres (Griewatz et al. 2020) und ein weiteres Supervisionsverständnis gegenüberstehen (Johnssohn 2020).

\subsection{Der Aus- und Wiedereinzug in die sozialen Organisationen}

Eine weitere Entwicklungslinie der deutschsprachigen Supervision ist die Abnabelung vom amerikanisch geprägten Modell der Vorgesetzten- (administrativen) Supervision. In Deutschland ist die „externe Supervision“ zum Standardmodell und Standardangebot in Sozialeinrichtungen geworden. Der Supervisor ist weder Vor-

\footnotetext{
2 Weigand schreibt von der „Psychologen-Regelung“ als einem Kampfbegriff, weil es schwer gewesen sei, diejenigen, ,die auf ihrer akademischen Vormachtstellung gegenüber der Sozialarbeit pochten, nun in den Supervisionsverband aufzunehmen“ (Weigand 2012, S. 20).
} 
gesetzter noch direkt in die Organisationsstruktur eingebaut, er ist in die Rolle eines autonomen Vertragspartners geschlüpft, was das Professionalisierungsprojekt der Supervision erheblich vorangetrieben hat. Auch die Einbindung in die Sozialarbeiterausbildung als Ausbildungssupervision (Praxisreflexion und Case-WorkUnterricht; vgl. Pühl 1994) hat sich stark relativiert. In den Studiengängen Sozialer Arbeit an den Fachhochschulen ist sie bis heute in verschiedenen Formen präsent. Als ,geschützter Raum“ ist sie aber zugleich marginalisiert und führt ein exterritoriales Nischendasein (Geißler-Pilz 2017, S. 22). Auch die sog. ,interne Supervision“ in Organisationen sozialer Arbeit ist von der Vorgesetzten- oder Leitungsrolle entbunden, aber z. B. über Stabs- und Fachstellen teilautonom an das Management gekoppelt und dient neben organisationsinternen Angeboten auch dem Einkauf und der Koordination externer Supervisoren (Becker 1994).

Die strukturelle Kopplung zwischen Supervision und Organisation bzw. ihr Wiedereintritt in die Sozialorganisationen wird durch den sog. „Dreieckskontrakt“ zwischen Auftraggeber, Supervisand und Supervisor geregelt. In ihm werden zwischen den Kontraktparteien Anliegen, Rahmen, Setting und Honorar vereinbart (Gröning 2016, S. 92 ff.). Eine genaue und sorgfältige Auftragsklärung gilt heute als Goldstandard professioneller Supervision (DGSv 2012, S. 20). Im „Dreieckskontrakt“ spiegelt sich nicht zufällig die Logik des ,,sozialhilferechtlichen Dreiecksverhältnisses“, in dem die Supervision sozusagen die Position des „Leistungserbringers“ einnimmt (s. oben). Damit ist Supervision zwar nicht mehr Teil einer (Sozial-) Organisation, aber Teil einer institutionellen Triade. Durch diesen Auszug aus der Organisation Sozialer Arbeit ist ihr explizit kontrollierender Aspekt im Wesentlichen durch die externe Unterstützung professioneller Selbstkontrolle ersetzt worden. Aus dieser Entwicklung ergab sich die Leitfrage, wie die Supervision als ein „hinzukommender Dritter“ eine wichtige triangulierende Rolle übernehmen und mit den unterschiedlichen Aufträgen und Erwartungen umgehen kann (Busse und Tietel 2018).

\subsection{Die Entdeckung der Organisation und die Begegnung mit der Organisationsberatung}

„Die Auseinandersetzung mit der Organisation stand schon 1968 ganz oben auf der Agenda“, so Leuschner und Weigand (2011, S. 51) aus der Perspektive von 2011. In den 90er-Jahren wird die Organisation zum dominierenden Thema. Nicht mehr allein „unser Gesicht dem Klienten zu(zuwenden) und unseren Rücken der Organisation“ (Hege und Weigand 1997, S. 5), ist Ausdruck eines gesteigerten Institutions- und Organisationsbewusstseins in der supervisorischen Community. Die professionelle Hilfearbeit ist über das Arbeitsbündnis mit dem Klienten hinaus kontextualisiert worden. Das Leid an der Bürokratie, die verwaltungslogische Einengung professioneller Autonomie, die gesellschaftlichen Normierungserwartungen an Hilfsbedürftige, die zunehmend auch ökonomisch eingrenzenden Zumutungen an soziale Organisationen, die Erfahrung schlechter oder guter Führung und die immer wieder erfahrbaren Grenzen institutionalisierter Hilfe wurden zu Quellen komplexer Reflexionsanliegen in Organisationen (Buchinger 1997; Pühl 2000). Teamsupervision (Weigand 1994a), Organisationberatung (Gotthardt-Lorenz 1989) bzw. Organisati- 
onssupervision (Gotthardt-Lorenz 2020), Leitungsberatung (Weigand 1994b), aber auch komplexe Beratungsdesigns, in denen Supervision neben anderen Formaten implementiert ist, sind Ausdruck eines erweiterten und komplexeren Supervisionsverständnisses (Heltzel und Weigand 2012).

Mit dieser Horizonterweiterung ist eine weitere Frage virulent geblieben: An wessen Tisch sitzt die Supervision in ihrer Gastrolle in den Organisationen? Wem man dienen soll, ist eine Frage, auf die man schon im Ursprungsland der Supervision gestoßen ist. Die Beziehung zur Organisation war und ist vor allem in der Sozialen Arbeit ambivalent. Man kann hierin bis heute eine generelle „Organisationsfeindlichkeit" der sozialen Arbeit als Ausdruck ihrer verfehlten (einseitigen) Professionalisierung der Helfer-Klienten-Interaktion sehen (Gschosmann 2017). Diese „Organisationsfeindlichkeit“ stellt sich in Supervisionen dann dem inzwischen gewachsenen triadischen Verständnis der Supervisoren entgegen oder fordert diese heraus.

Aber nicht nur die Organisation ist eine Herausforderung für die Supervision gewesen - sondern auch die Konfrontation mit der Organisationsberatung. Durch das stärker gewordene Organisationsbewusstsein auch in den sozialen Dienstleistungsorganisationen hat sie sich dort als „Nebenbuhlerin“ der Supervision etabliert. Aus dem For-Profit-Bereich stammend - entstanden im Zuge der Human-Relations-Bewegung und im Kontext der Unternehmensberatung (vgl. v. Ameln et al. 2009) -, ist sie sozusagen von oben in die Organisation eingestiegen. Damit hatte sich die Frage der Zuständigkeits- und Marktkonkurrenz für die Supervision mit einem anderen Format zum ersten Mal scharf gestellt. Daraus resultierte zunächst die pragmatische wie theoretische Frage nach der Grenzziehung: Ist Supervision nicht immer auch Organisationsentwicklung? Aber wann ist sie schon Organisationsberatung? Es galt und gilt bis heute, auch im Beratungsalltag den Unterschied zwischen reflexiver Beratung in und Beratung von Organisationen im Blick zu haben, das heißt, immer wieder zu klären, was das angemessene Format und was die angemessene Fokussierung auf die Person, die Rolle, das Team und die Organisation ist. Eine mögliche umfassende Antwort ist das Konzept einer eigenständigen „Organisationssupervision“, die jegliches professionelles und berufliches Handeln als organisationales Handeln konzeptualisiert (Gotthardt-Lorenz 2020).

Die Frage der Grenzziehung zwischen beiden Beratungsformaten birgt aber noch eine tiefer gehende in sich - die nach dem Verhältnis von Profession und Organisation (Buer 2010). Für die Supervision ergibt sich daraus die Leiffrage, wie sie sich selbst immer wieder zwischen Humanität oder Funktionalität positioniert (Buer 2010).

\subsection{Vom psychotherapeutischen Blick zur Kompetenzorientierung}

Ein wichtiger Nebenaspekt vor allem der Gegenstandserweiterung durch die Organisationsperspektive ist eine Erweiterung des konzeptuellen Blicks gewesen. Er hat die frühere Konzentration auf die Helfer-Klienten-Beziehung in der Supervision und die globale Kritik an der Institution und Organisation relativiert. Die begriffliche Klammer dafür ist die Einsicht, Supervision habe es immer mit der Triade von Person, Rolle und Institution (Organisation) zu tun (Weigand 1985), die sogenannte 
Primäraufgabe der Organisation ist ein Fixpunkt sozialarbeiterischen, professionellen resp. beruflichen Handelns und damit supervisorischer Reflexion. Damit findet auch die primäre Orientierung an psychotherapeutischen Konzepten (durch den sog. „Psychoboom“ der 1970er-Jahre) eine Art soziologische Korrektur. Es kam zu einer Expansion des Horizonts supervisorischen Wissens, weil die Komplexität des supervisorischen Gegenstands zunahm bzw. man seiner Komplexität gewahr wurde.

Integrative Konzepte (Schreyögg 1991; Petzold 1998) und die Rezeption systemtheoretischer Ansätze haben zu dieser Perspektivenerweiterung wesentlich beigetragen (Ebert 2001; Schiersmann und Thiel 2012). Auch klassisch psychotherapeutische Brillen nehmen Organisationsphänomene etwa als „Psychoanalyse der Organisation“ in den Blick (Lohmer und Möller 2014; Giernalczyk und Möller 2018). Im Übrigen: Auch wenn ein Gutteil der Supervisoren ihre Identität bis heute immer noch auf dem Hintergrund psychotherapeutischer Schulen definieren, so geht die Frage, was Supervisoren wissen und können müssen, eindeutig in Richtung Schulen übergreifender und methodenintegrierender Kompetenzprofile. Die Orientierung an kompetenzbasierter Supervision läutete im europäischen (Judy und Knopf 2016; Busse und Jahn 2020) wie auch im internationalen Supervisionsdiskurs eine neue Ära für das 21. Jahrhundert ein, die sehr eng mit dem Wirkungsnachweis von Supervision verbunden ist (Falender und Shafranske 2007, 2012).

\subsection{Die ,Verarbeitsweltlichung“ der Supervision}

Bereits in den 90er-Jahren eingeleitet, kommt es ab 2000 zu einem weiteren Schritt der ,,horizontalen“ und der ,,vertikalen Expansion“ der Supervision (Kühl 2006): Sie wendet sich globalen Phänomenen und Folgen des Strukturwandels der Arbeitswelt zu. Dies führt über ihren Berufs- und Organisationsbezug hinaus zu einer weiteren Ausdehnung ihres reflexiven Terrains (Hausinger 2008; Haubl et al. 2013a).

Die allgemeinen seit den 80er-Jahren soziologisch beschriebenen Merkmale der „Zweiten Moderne“ machen sich in der Arbeitswelt als die Zunahme von Wissensarbeit, durch die Konfrontation mit Entgrenzungs- und Subjektivierungsphänomenen in Leben und Beruf, die Digitalisierung von Arbeit und Leben etc. bemerkbar. Arbeit muss notorisch unter Bedingungen von Nichtwissen und Ungewissheit verrichtet werden, sie wird zunehmend auch Beziehungsarbeit und unter der Ausweitung des Dienstleistungssektors zur Interaktionsarbeit, in der ko-konstruktiv an der Sache gearbeitet wird und Emotions- und Gefühlsregulation zum Kompetenzstandard werden. Arbeitskontexte (vor allem Organisationen) büßen dabei ihre haltende und bindende Funktion weitgehend ein (Tietel 2003) und werden zur VUCA-Welt (Volatilität, Unsicherheit, Komplexität und Ambiguität; vgl. Mack et al. 2015). Ambiguitätstoleranz und Ambivalenzfähigkeit werden zu neuen Schlüsselkompetenzen. Das stellt Reflexivität und reflexive Selbststeuerung sozusagen auf Dauer und empfiehlt der Supervision „Arbeit“ als generelles Reflexionsfeld an (Buchinger 1999). Schaut man mit einer naiven modernisierungstheoretischen Brille nur auf die Chancen dieser gesellschaftlichen Veränderungen, dann erscheinen die Unterschiede zwischen NonProfit und Profit-Sektor zunehmend als überholt, unerheblich und egalisiert (vgl. Belardi 2018, S. 35), etwa nach dem Motto: „Dort wo reflektiert wird, sind wir schon immer dabei“. 
Die eigentliche Herausforderung lag und liegt aber darin, sich auch mit den Schattenseiten und Risiken dieser Entwicklung zu befassen - mit Veränderungserschöpfung, mit neuen Entfremdungsformen durch Selbstoptimierung, mit den psychosozialen Kosten wie Burnout, mit nachlassender Bindungsfähigkeit und -willigkeit vor allem in und an Organisationen (Busse 2018) etc. So hat sich Supervision zu einem Spiegel und Seismographen der ,riskanten Arbeitswelt“ auf den Weg gemacht und damit an ein Selbstverständnis angeknüpft, Supervision auch als eine Art kritische Feldforschung zu verstehen (Haubl und Voß 2011; Haubl et al. 2013b). ${ }^{3}$ Durch die „Verarbeitsweltlichung“ der Supervision ist diese mit der Leitfrage konfrontiert, wie sie sich in einer Welt, in der Reflexivität en vogue ist und zugleich einer Verwertungslogik unterliegt, als kritische Reflexion des Reflexionsimperativs positionieren kann.

Diese Entwicklungslinie der Supervision enthält noch eine weitere Pointe: Die skizzierten Veränderungen der Arbeitswelt haben den Sozialsektor als dessen Ökonomisierung ebenfalls erreicht (Hammerschmidt et al. 2017). Die Einführung der privatwirtschaftlich orientierten „Neuen Steuerung“ in den öffentlichen Verwaltungen seit Mitte der 90er-Jahre brachten Sozialmanagement, Controlling und andere betriebswirtschaftlich ausgerichtete Konzepte in die sozialen Dienstleistungsorganisationen. Die ursprünglich emanzipatorische Kritik an der starren Bürokratie von Sozialorganisationen wurde von einer konservativen und neoliberalen Kritik an sozialen Organisationen überlagert, wenn nicht gar überholt. Diese war durchschlagender als jede emanzipatorische Hoffnung von unten. Für die Beschäftigten in vielen sozialen Einrichtungen hieß das zunehmend, nicht nur an die Grenzen des professionell noch Tragbaren, sondern auch an jene der erwerbsmäßig noch Ertragbaren (Belastung) zu gehen. Es wurde deutlicher, dass es auch im Sozialsektor darum geht, Arbeit nicht nur gut (professionell) zu machen, sondern auch gute Arbeit zu haben (Kunkel und Tietel 2018). Zu dem klassischen Widerspruch zwischen Profession und Organisation (s. oben) ist der zwischen Profession und Ökonomie hinzugetreten. Das führte dazu, dass ,,die Reflexion der Beziehung zum Klienten (...) im Vergleich mit den unmittelbaren Arbeitsbedingungen in den Hintergrund rückt" (Fritsch 2011, S. 71). Soziale Arbeit ist nicht mehr nur Arbeit am und für Klienten, sondern auch Erwerbsarbeit für Sozialarbeitende.

\subsection{Supervision trifft auf Coaching}

Damit nähern wir uns dem bislang letzten Kapitel der Wandlungen, die die Supervision an der Seite und zugleich in Distanzierung zur Sozialen Arbeit durchgemacht hat: Die Veränderungen der Arbeitswelt haben, wie eben beschrieben, Arbeit vor allem. Die Veränderung der Rolle des Subjekts von der Störgröße hin zu einem verwertbaren Springquell von Produktion und Produktivität hat im For-Profit-Bereich einen immensen Reflexions- und riesigen Beratungsbedarf entstehen lassen. Durch ihn wurde seit den 90er-Jahren ein Coachingboom ausgelöst (Böning 2005; Fellermann 2011). Die Versuche und Offerten, Supervision auch in der Wirtschaft zu etablieren

\footnotetext{
${ }_{3}^{3}$ Das Forschungsprojekt „Arbeit und Leben in Organisationen“ 2008-2011 wurde in Kooperation der DGSv, des Sigmund-Freud-Instituts Frankfurt a.M. und der Universität Chemnitz durchgeführt.
} 
(s. oben), wurden von einem exzessiven wie expandierenden Coachingmarkt konterkariert und überrannt. Hier geht es auch um Konkurrenz und Zuständigkeitsfragen, aber mehr noch um die Identität und das eigene Selbstverständnis der Supervision bzw. der Supervisoren.

Die Herkunft des Coachings aus einem anderen „Ursprungsland“ (Sport, Training, Mentoring an Universitäten und als Beratung von Führungskräften; vgl. v. Schumann 2014, S. 3) macht dieses kulturell und sachlogisch zunächst mit einer Welt der Effizienz, der Verwertungslogik, der strikteren Ergebnis- und Zielorientierung kompatibler. Aber: Coaching ist inzwischen selbst in die Welt des Ursprungslandes der Supervision eingedrungen. In sozialwirtschaftlichen Organisationen ist es durch die starke Orientierung an Organisations- und Führungsfragen Usus geworden, dass das Führungspersonal Coaching und die an der Basis arbeitenden Professionals weiterhin Supervision erhalten. Das ist mehr als eine stille Hierarchisierung der Beratungsformate. So kann man fast auf die Idee kommen, die Soziale Arbeit bräuchte eher Coaching als Supervision, da diese ja Coaching für helfende Berufe sei (vgl. Loebbert 2016).

Mit Supervision und Coaching treffen nicht nur Beratungsformate unterschiedlichen Ursprungs und unterschiedlicher Prägung aufeinander, sondern unterschiedliche arbeitsweltliche Handlungslogiken. Auf der einen Seite haben wir das Primat komplexer professioneller Beziehungsarbeit und -reflexion (Supervision) und auf der anderen Seite das Primat der eher linearen produkt-, ziel- und ergebnisorientierten Arbeit (Coaching), die sich den beiden Beratungsformaten eingeschrieben haben. Dies entspricht der Differenz zwischen kritischer Kontextreflexion und an Performanz orientierter Handlungspragmatik. Dahinter stehen auch unterschiedliche Grundwerte - hier die Gemeinwohlorientierung mit ihrem emanzipatorischen Anspruch und dort die Logik von Verwertungs- und Steigerungslogik. Kurz gesagt, das entspricht der Differenz zwischen Moral und Markt. Aber lässt sich dieser Widerspruch wirklich auf die einfache Formel bringen: „Coaching heißt: Fit-Machen. Supervision heißt: Zum Nachdenken bringen “, wie es Buer (2005, S. 282) seinerzeit provozierend und pointiert zum Ausdruck gebracht hat?

Entsprechend gab es in den letzten beiden Dekaden eine ganze Reihe von Versuchen der Supervision, sich dem Coaching gegenüber zu positionieren: Am Anfang standen eher schroffe Abwehr und Entgegensetzung. Coaching sei der Versuch „(l)ieber mit den Wölfen (zu) heulen als mit den Schafen (zu) blöken?“ (Bauer 2004). „Die mehrdimensionale Interpretations- und Arbeitsweise wie der fachlich-ethische Blick ist im Coaching nicht vorhanden“ (Buer 2010, S. 59). Das wechselte mit dem Plädoyer für „,ein friedliches Zusammenspiel“ der Formate (Buer 2005). Das wurde wiederum gefolgt von dem Versuch, Coaching in das qualitativ anspruchsvollere (höherwertige) Format der Supervision zu integrieren. Werbewirksam hieß es dann 2009 in einer Offensive der DGSv: „Mein Coach ist Supervisor. Und Ihrer?“. Coaching sollte aber nur eine Methode sein, Supervision ein Konzept (Weigand 2002) usw. usf.

Inzwischen sind diese Abwehr-, Assimilations- und Integrationsversuche eher dem Versuch gewichen, die weitere Professionalisierung von Supervision und Coaching voranzutreiben (Hausinger und Volk 2013). Das wird von sichtbaren Professionalisierungsbemühungen des Coachings begleitet - durch seine starke Forschungs- 
orientierung, durch Fragen nach seinem Status als autonom handelnde Profession (Fietze 2011; Schmidt-Lellek 2004) und nach seiner gesellschaftlichen wie politischen Wirksamkeit (Böning und Strikker 2014, S. 495). Die DGSv, die seit 2017 auch Coaching in ihrem Namen trägt, ist als Gründungsmitglied am sog. „Roundtable“ unterschiedlicher Coachingverbände (RTC) beteiligt ${ }^{4}$, wo es um Standards professioneller arbeitsweltlicher Beratung geht. Damit sind Gleichheit und Differenz von Supervision und Coaching behauptet. Aber worin bestehen das Verbindende und das Trennende beider Formate? Wird es in Zukunft eine gemeinsame Professionalisierungsgeschichte geben, in der diese Gleichheit und Differenz Bestand haben und gelebt werden können? Oder wird die Supervision, die aus ihrem Ursprungsland der Sozialen Arbeit auszog, in dieses als Coaching zurückkehren? Was bleibt vom genuin Supervisorischen in einer alles übergreifenden VUCA-Welt?

Diese Fragen bestimmen die aktuelle Debatte in der Community der Supervisoren (Bentele und Fellermann 2012). Es stehe eine ,längst überfällige grundsätzliche Diskussion über professionsethische Grundlagen von bzw. für Supervision und Coaching“ an (Austermann 2019, S. 6). Sie hat z. B. als Debatte ,über die strategische Ausrichtung der DGSv“ längst begonnen, vor allem ,über Moral und Markt, Beruf und Profession, Alt und Jung“ (Austermann et al. 2018).

\section{Supervision zwischen Gegenstands-, Zuständigkeits- und Kompetenzerweiterung}

Blicken wir auf die skizzierten Entwicklungslinien der deutschen bzw. deutschsprachigen Supervision zurück, so ergibt sich ein widersprüchliches Bild. Es ist komplexer als die seinerzeit von Kühl (2006) diagnostizierte ,horizontale und vertikale Expansion“ der Supervision. Wir haben es eher mit der Gleichzeitigkeit und Überblendung von drei Erweiterungsdynamiken zu tun:

1. Mit einer Gegenstanderweiterung: Supervision hat sich als ein Anleitungsformat zur Einübung und Reflexion methodischen Handelns in der Sozialen Arbeit zunehmend kontextualisiert und erweitert. Teams, Organisationen, Institution, der gesellschaftliche, politische, kulturelle und arbeitsweltliche Kontext sind als Rahmungen und strukturelle Bedingungen der klassischen Helfer-Klient-Beziehung immer reflexionsrelevanter geworden. Das hat den Blick über das klassische Arbeitsbündnis hinaus enorm geweitet. Aber allein mit dieser gern als Multiperspektivität apostrophierten Gegenstandserweiterung hätte Supervision noch im Geviert der Sozialen Arbeit verbleiben können.

2. Mit einer Zuständigkeitserweiterung: Das zentrale Beratungsfeld für Supervisoren sind nach wie vor Einrichtungen und Handlungsfelder der Sozialen Arbeit. Aber Supervision ist nicht mehr Sozialarbeitssupervision. Ihre Offerten gehen weit darüber hinaus und gelten als ein Angebot für weitere professionalisierte und reflexionsbedürftige Berufstätigkeiten. Erst dies hat ihr Zuständigkeitsüberschnei-

\footnotetext{
4 Vgl. das Positionspapier des RTC: http://www.roundtable-coaching.eu/wp-content/uploads/2015/03/ RTCProfession-Coach-2015-03-19-Positionspapier.pdf, 02.09.2019.
} 
dungen und Abgrenzungsprobleme mit konkurrierenden Beratungsformaten (vor allem mit der Organisationsberatung und dem Coaching) beschert. Das daraus resultierende Unschärferegime an den Grenzen ihrer Zuständigkeit hat sie im Übrigen mit der Sozialen Arbeit gemein.

3. Mit einer Kompetenzerweiterung: Im Zuge dieser eben genannten Erweiterungen (Expansionen) ist es zwangsläufig auch zu einer Erweiterung supervisorischer Kompetenz gekommen. Die primäre Bindung an psychotherapeutische Schulen hat abgenommen und bleibt zugleich als eine Vielfalt von diagnostischen und interventiven Zugängen bestehen. Über das rein quantitative Anwachsen konzeptueller Brillen und Wissensformen hat sich eine Suchbewegung nach Kernkompetenzen eingestellt, die die Supervision als reflexionsorientierte Prozessberatung wiederum mit anderen Beratungsformaten teilt und ihr vermutlich nicht als Alleinstellungsmerkmal gereichen wird.

Es macht Sinn, diese drei Erweiterungsdimensionen der Supervision zumindest analytisch zu unterscheiden. Real werden sie sich weiter überschneiden und überblenden. Das macht es verständlich, warum das Bestimmen des „Supervisorischen“ bis heute so mühsam ist (Weigand 2007). Für die weitere Professionalisierung der Supervision wird die notwendige Grenz- und Begrenzungsarbeit aber nicht hinreichend sein. Entscheidender ist etwas anderes: Supervisoren sind wie ihre Kunden und Klienten mit widersprüchlichen, paradoxen, multirationalen und hybriden Anforderungen und Logiken - zwischen Interaktion und Bürokratie, Profession und Organisation, Emanzipation und Anpassung, Markt und Moral etc. - konfrontiert. Nicht allein die Grenzziehungen, sondern der Umgang mit diesen ist die eigentliche Bewährungsprobe ihrer Professionalisierung. Das teilt sie wiederum mit ihrem Ursprungsland - der Sozialen Arbeit.

Funding Open Access funding enabled and organized by Projekt DEAL.

Open Access Dieser Artikel wird unter der Creative Commons Namensnennung 4.0 International Lizenz veröffentlicht, welche die Nutzung, Vervielfältigung, Bearbeitung, Verbreitung und Wiedergabe in jeglichem Medium und Format erlaubt, sofern Sie den/die ursprünglichen Autor(en) und die Quelle ordnungsgemäß nennen, einen Link zur Creative Commons Lizenz beifügen und angeben, ob Änderungen vorgenommen wurden.

Die in diesem Artikel enthaltenen Bilder und sonstiges Drittmaterial unterliegen ebenfalls der genannten Creative Commons Lizenz, sofern sich aus der Abbildungslegende nichts anderes ergibt. Sofern das betreffende Material nicht unter der genannten Creative Commons Lizenz steht und die betreffende Handlung nicht nach gesetzlichen Vorschriften erlaubt ist, ist für die oben aufgeführten Weiterverwendungen des Materials die Einwilligung des jeweiligen Rechteinhabers einzuholen.

Weitere Details zur Lizenz entnehmen Sie bitte der Lizenzinformation auf http://creativecommons.org/ licenses/by/4.0/deed.de.

\section{Literatur}

Althoff, M. (2018). Fallverständnis in der Sozialen Arbeit und seine Relevanz für Fallsupervision. Forum Supervision, 51, 6-19. https://doi.org/10.4119/fs-2328. 
v. Ameln, F., Kramer, J., \& Stark, H. (2009). Organisationsberatung beobachtet. Hidden Agendas und Blinde Flecke. Wiesbaden: Springer VS.

Austermann, F. (2019). Zu den demokratischen, emanzipatorischen und intentionalen Wurzeln der Profession Supervision am Beispiel von Cora Baltussens Beratungsverständnis. Forum Supervision, 52, 6-16. https://doi.org/10.4119/fs-2320.

Austermann, F., et al. (2018). „Wesentlich, dass zukünftig die Orientierung an der Praxis der Supervision den Ton angibt." Eine Debatte über die strategische Ausrichtung der DGSv. Journal Supervision, 4, $12-19$.

Bauer, A. (2004). „Lieber mit den Wölfen heulen als mit den Schafen blöken?“ Anmerkungen zur Kontroverse Supervision und Coaching. In F. Buer \& G. Stiller (Hrsg.), Die flexible Supervision. Herausforderungen - Konzepte - Perspektiven. Eine kritische Bestandsaufnahme (S. 121-142). Wiesbaden: Springer VS.

Becker, P. (1994). Externe Supervision - Interne Supervision. In H. Pühl (Hrsg.), Handbuch der Supervision (Bd. 2, S. 344-352). Berlin: Edition Marhold.

Belardi, N. (1992). Supervision. Von der Praxisberatung zur Organisationsentwicklung. Paderborn: Junfermann.

Belardi, N. (2018). Supervision und Coaching. Grundlagen, Techniken, Perspektiven. München: C.H. Beck.

Bentele, M., \& Fellermann, J. (Hrsg.). (2012). Womit Supervision und Coaching zu tun haben werden. Schlaglichter auf Veränderungen in Gesellschaft, Arbeit und Beratung. Kassel: University Press.

Blinkert, B., \& Huppertz, N. (1974). Der Mythos der Supervision. Kritische Anmerkungen zu Anspruch und Wirklichkeiten. Neue Praxis, 2, 117-132.

Böning, U. (2005). Coaching: Der Siegeszug eines Personalentwicklungs-Instrumentes - Eine 15-JahresBilanz. In C. Rauen (Hrsg.), Handbuch Coaching (S. 21-55). Göttingen: Hogrefe.

Böning, U., \& Strikker, F. (2014). Ist Coaching nur Reaktion auf gesellschaftliche Entwicklungen oder auch Impulsgeber? Organisationsentwicklung, Supervision, Coaching, 21(4), 483-496.

Brutzer de Palma, N. (2019). Wie, wo und für wen arbeiten Sie? Die Ergebnisse der Mitgliederbefragung „Ihre Tätigkeit im Markt 2018“. Journal Supervision, 1, 30-33.

Buchinger, K. (1997). Supervision in Organisationen. Heidelberg: Carl-Auer.

Buchinger, K. (1999). Die Zukunft der Supervision. Aspekte eines neuen „Berufs “. Heidelberg: Carl-Auer.

Buer, F. (2005). Coaching, Supervision und die vielen anderen Formate. Ein Plädoyer für ein friedliches Zusammenspiel. Organisationsentwicklung, Supervision, Coaching, 12(3), 278-297.

Buer, F. (2010). Gefährdet Organisation Profession? In A. Schreyögg \& C. Schmidt-Lellek (Hrsg.), Die Organisation in Supervision und Coaching (S. 41-60). Wiesbaden: Springer VS.

Busse, S. (2009). Supervision between critical reflection and practical doing. Journal of Social Work Practice, 23(2), 159-175.

Busse, S. (2018). Bindung(en) in der Arbeitswelt. Gruppe. Interaktion. Organisation, 49(4), 305-317.

Busse, S. (2021, in press). A German Perspective on Supervision: Supervision between maintenance of, emancipation from or abnegation of the origins of social work? In L. Engelbrecht \& K. O'Donoghue (Hrsg.), International Handbook of Supervision of Social Work. London, New York: Routledge.

Busse, S., \& Jahn, R. (2020). Excellence in supervision and coaching training: considerations. European Journal of Supervision and Coaching, 4, 59-64.

Busse, S., \& Tietel, E. (2018). Mit dem Dritten sieht man besser: Triaden und Triangulierung in der Beratung. Göttingen: Vandenhoeck \& Ruprecht.

DGSv (Hrsg.). (2012). Supervision - ein Beitrag zur Qualifizierung beruflicher Arbeit. Köln: DGSv.

DGSv (2020). Mitgliederbefragung (16.9.2020). https://www.dgsv.de/?s=Mitgliederbefragung. Zugegriffen: 21. Febr. 2021.

Diebäcker, H. (1995). Chancen und Risiko der Personalentwicklung mit Supervision in Profit-Unternehmen. Supervision, Zeitschrift für berufsbezogene Beratung, Sonderheft, 21-37.

Ebert, W. (2001). Systemtheorien in der Supervision. Wiesbaden: Springer VS.

Engelbrecht, L., \& O'Donoghue, K. (Hrsg.). (2021). International handbook of supervision of social work. London, New York: Routledge.

Falender, C. A., \& Shafranske, E. P. (2007). Competence in competency-based supervision practice: construct and application. Professional Psychology Research and Practice. https://doi.org/10.1037/07357028.38.3.232.

Falender, C. A., \& Shafranske, E. P. (2012). The importance of competency-based clinical supervision and training in the twenty-first century: why bother? Journal of Contemporary Psychotherapy, 42(3), 129-137. https://doi.org/10.1007/s10879-011-9198-9. 
Fellermann, J. (Hrsg.). (2011). Supervision und Coaching auf dem Beratungsmarkt. Eine explorative Studie als Beitrag zur Marktforschung. Göttingen: Vandenhoeck \& Ruprecht.

Fietze, B. (2011). Profilbildung und Strukturkonkurrenz. Zur Bedeutung der Distinktionsstrategien im Professionalisierungsprozess von Supervision und Coaching. In E.-M. Graf, Y. Aksu, I. Pick \& S. Rettinger (Hrsg.), Beratung, Coaching, Supervision. Multidisziplinäre Perspektiven vernetzt (S. 23-36). Wiesbaden: Springer VS.

Fritsch, J. (2011). Supervision 2008. Schlaglichter auf Veränderungen in der Profession. In R. Haubl \& G. G. Voß (Hrsg.), Riskante Arbeitswelt im Spiegel der Supervision. Eine Studie zu den psychosozialen Auswirkungen spätmoderner Erwerbsarbeit (S. 68-74). Göttingen: Vandenhoeck \& Ruprecht.

Fürstenau, P. (1995). Supervision auf dem steinigen Weg zu neuen Arbeitsfeldern. Supervision, Zeitschrift für berufsbezogene Beratung, Sonderheft, 10-21.

Geißler-Piltz, B., (2017). Statt Marginale: Integration von Supervision im Studium der Sozialen Arbeit. Supervision. Mensch Arbeit Organisation, 35(2), 16-26.

Giernalczyk, T., \& Möller, H. (2018). Entwicklungsraum: Psychodynamische Beratung in Organisationen. Göttingen: Vandenhoeck \& Ruprecht.

Gotthardt-Lorenz, A., (1989). Organisationsberatung. Hilfe und Last für die Sozialarbeit. Freiburg: Lambertus.

Gotthardt-Lorenz, A. (2020). Organisationssupervision - Ein Konzept. Göttingen: Vandenhoeck \& Ruprecht.

Gotthardt-Lorenz, A., \& Walther, I. (1998). Berufs- und Arbeitsfeldorientierung - der Blickwinkel, der Supervision zur Supervision macht. Supervision, Österreichische Vereinigung Für Supervision, 2 , $7-11$.

Griewatz, H.-P., Jahn, R., Krüger, P., \& Leser, C. (2020). Gegen begriffliche Unschärfe. Warum wir die Unterscheidung in Supervision und Coaching für die Professionalisierung von Supervision für unerlässlich halten. Supervision. Mensch Arbeit Organisation, 3, 4-9.

Gröning, K. (2016). Sozialwissenschaftlich fundierte Beratung in Pädagogik, Supervision und Sozialer Arbeit. Gießen: Psychosozial-Verlag.

Gschosmann, A. (2017). Das Unbehagen mit dem Dreieckskontrakt. Überlegungen zu einer Konstante in der Teamsupervision von Sozialorganisationen. Supervision, Mensch Arbeit Organisation, 2, 27-36.

Hammerschmidt, P., Sagebiel, J., \& Yollu-Tok, A. (Hrsg.). (2017). Die Soziale Arbeit im Spannungsfeld der Ökonomie. Schriftenreihe Soziale Arbeit der Fakultät für angewandte Sozialwissenschaften der Hochschule München.

Haubl, R., \& Voß, G. G. (Hrsg.). (2011). Riskante Arbeitswelt im Spiegel der Supervision. Eine Studie zu den psychosozialen Auswirkungen spätmoderner Erwerbsarbeit. Göttingen: Vandenhoeck \& Ruprecht.

Haubl, R., Hausinger, B., \& Voß, G. G. (2013a). Riskante Arbeitswelten: Zu den Auswirkungen moderner Beschäftigungsverhältnisse auf die psychische Gesundheit und die Arbeitsqualität. Frankfurt/M.: Campus.

Haubl, R., Voß, G. G., Alsdorf, N., \& Handrich, C. (Hrsg.). (2013b). Belastungsstörung mit System. Göttingen: Vandenhoeck \& Ruprecht.

Hausinger, B. (2008). Supervision: Organisation - Arbeit - Ökonomisierung: Zur Gleichzeitigkeit des Ungleichzeitigen in der Arbeitswelt (Arbeit und Leben im Umbruch). Mering: Rainer Hamp.

Hausinger, B., \& Volk, T. (2013). Erneuerung statt Imagepflege. In DGSv (Hrsg.), Sonderpublikation zum Journal Supervision. 4/2013. Köln: DGSv.

Hege, M. (1979). Engagierter Dialog. Ein Beitrag zur sozialen Einzelhilfe (2. Aufl.). München, Basel: Ernst Reinhardt.

Hege, M. (2011). Supervision im Prozess der Veränderung beruflicher Arbeit im Allgemeinen Sozialdienst (ASD). Forum Supervision. 37, 58-71. https://doi.org/10.4119/fs-2147.

Hege, M., \& Weigand, W. (1997). „Ich sitze immer dazwischen und bemühe mich, auch dort zu bleiben“. Marianne Hege im Gespräch mit Wolfgang Weigand. Supervision. Zeitschrift für berufsbezogene Beratung, 31, 4-16.

Heltzel, R., \& Weigand, W. (2012). Im Dickicht der Organisation: komplexe Beratungsaufträge verändern die Beratungsrolle. Göttingen: Vandenhoeck \& Ruprecht.

Huppertz, N. (1975). Supervision. Analyse eines problematischen Kapitels der Sozialarbeit. Neuwied: Luchterhand.

Johnssohn, M. (2020). Irgendwo im Nirgendwo - da muss sie liegen, die Zukunft der Supervision. Supervision. Mensch Arbeit Organisation, 3, 10-15.

Judy, M., \& Knopf, W. (Hrsg.). (2016). In the mirror of competences. Wien: facultas. 
Kühl, S. (2006). Die Supervision auf dem Weg zur Profession? Professionalisierung im Spannungsfeld zwischen Expansionsbestrebung und Selbstbescheidung. Organisationsberatung, Supervision, Coaching, 13(1), 5-19.

Kunkel, R., \& Tietel, E. (2018). „Gute Arbeit“ als professions- und arbeitspolitisches Projekt. Positionen, Beiträge zur Beratung der Arbeitswelt, Bd. 1. Kassel: University Press.

Leuschner, G., \& Diebäcker, H. (1995). Briefwechsel: Supervision in Profit-Unternehmen. Supervision. Zeitschrift für berufsbezogene Beratung, Sonderheft, 37-47.

Leuschner, G., \& Weigand, W. (2011). Wege zur Professionalisierung - Über die Anfänge der Supervision in Deutschland. Forum Supervision, 37, 38-58. https://doi.org/10.4119/fs-2146.

Lippenmeier, N. (2011). Der Entwicklungsprozess der Supervision in Deutschland und wie es zur Gründung der DGSv kam. Forum Supervision, 37, 8-16. https://doi.org/10.4119/fs-2141.

Loebbert, M. (2016). Wie Supervision gelingt. Supervision als Coaching für helfende Berufe. essentials. Berlin: Springer.

Lohl, J. (2019). „... und ging ins pralle Leben“. Facetten der Sozialgeschichte der Supervision. Göttingen: Vandenhoeck \& Ruprecht.

Lohmer, M., \& Moller, H. (2014). Psychoanalyse in Organisationen: Einführung in die psychodynamische Organisationsberatung. Psychoanalyse im 21. Jahrhundert. Stuttgart: Kohlhammer.

Lowy, L. (1983). Social work supervision: from models toward theory. Journal of Education for Social Work, 19(2), 55-62.

Mack, O., Khare, A., Kramer, A., \& Burgartz, T. (Hrsg.). (2015). Managing in a VUCA world. Heidelberg: Springer.

Pattar, A.K. (2012). Sozialrechtliches Dreiecksverhältnis - Rechtsbeziehungen zwischen Hilfsbedürftigen, Sozialhilfeträgern und Einrichtungsträgern. SozialRecht aktuell. Zeitschrift für Sozialberatung, $3,85-132$.

Petzold, H. (1998). Integrative Supervision, Meta-Consulting, Organisationsentwicklung: Ein Handbuch für Modelle und Methoden reflexiver Praxis. Paderborn: Junfermann.

Pühl, H. (1994). Supervision in der (Fach-) Hochschul-Ausbildung. In H. Pühl (Hrsg.), Handbuch der Supervision (Bd. 2, S. 406-418). Berlin: Marhold.

Pühl, H. (Hrsg.). (2000). Supervision und Organisationsentwicklung. Opladen: Leske + Budrich.

Schiersmann, C., \& Thiel, H. U. (2012). Beratung als Förderung von Selbstorganisationsprozessen: Empirische Studien zur Beratung von Personen und Organisationen auf der Basis der Synergetik. Göttingen: Vandenhoeck \& Ruprecht.

Schmidt-Lellek, C. J. (2004). Anmerkungen zur Professionalisierung des Coachings auf dem Hintergrund des klassischen Professionsbegriffs. Organisationsentwicklung, Supervision, Coaching, 11(2), $183-192$.

Schreyögg, A. (1991). Supervision. Ein integratives Modell. Paderborn: Junfermann.

v. Schubert, H. (1999). Unternehmenskultur in der freien Wohlfahrpflege - Fragen eines Mitarbeiters an den Träger. System Familie, 12, 109-119.

v. Schumann, K. (2014). Coaching im Aufwind. Professionelles Business-Coaching: Inhalte, Prozesse, Ergebnisse und Trend. Wiesbaden: Springer VS.

Stenzel, H. (2019). Louis Lowy - erlebte Geschichte(n) als Entwicklungsimpuls der Supervision in Deutschland. Forum Supervision, 52, 17-32. https://doi.org/10.4119/fs-2321.

Tietel, E. (2003). Emotion und Anerkennung in Organisationen. Wege zu einer triangulären Organisationskultur. Münster: Lit.

Weigand, W. (1985). Des Supervisors Gang in die Organisation. Supervision, Zeitschrift für berufsbezogene Beratung, 7, 1-8.

Weigand, W. (1993). Die Faszination des Geldes und des fremden Feldes - Supervision in Wirtschaftsunternehmen. Supervision, Zeitschrift für berufsbezogene Beratung, 24, 3-11.

Weigand, W. (1994a). Teamsupervision: Ein Grenzgang zwischen Supervision und Organisationsberatung. In H. Pühl (Hrsg.), Handbuch der Supervision (Bd. 2, S. 112-131). Berlin: Marhold.

Weigand, W. (1994b). Leitungsberatung. In H. Pühl (Hrsg.), Handbuch der Supervision (Bd. 2, S. 406-418). Berlin: Marhold.

Weigand, W. (2002). Coaching eine Methode - Supervision ein Konzept. In L. v. Kessel \& J. Fellermann (Hrsg.), Supervision und Coaching in europäischer Perspektive. Beiträge der ANSE-Konferenz 2000 (S. 45-54). Köln: Association of National Organisations for Supervision in Europe (ANSE).

Weigand, W. (2007). Das eigentlich Supervisorische - Eine Einführung in dieses Heft. Supervision. Mensch Arbeit Organisation. Die Zeitschrift für Berater/innen, 25(2), 4-5.

Weigand, W. (2012). Die DGSv in ihren (historischen) Spannungsfeldern. Forum Supervision, 39, 18-28. https://doi.org/10.4119/fs-2112. 
Wieringa, C.F. (1990). Entwicklungsphasen der Supervision (1860-1950). Supervision, Zeitschrift für berufsbezogene Beratung, 18, 36-44.

Yuen-han Mo, K., O’Donoghue, K., Yuh-ju Wong, P., \& Tsui, M. (2020). The historical development of knowledge in social work supervision: Finding new directions from the past. International Social Work. https://doi.org/10.1177/2F0020872819884995.

Prof. Dr. Stefan Busse Prof. Dr. rer. nat. habil., Dipl.-Psych., Fakultät Soziale Arbeit der Hochschule Mittweida, Studiengangleiter der Zertifikatstudienganges „Supervision und Coaching“ und an der Hochschule Mittweida, wissenschaftlicher Direktor des Institutes für „Kompetenz, Kommunikation und Sprachen“ (IKKS), Ausbildungsleitung bei Basta Fortbildungsinstitut für Supervision und Coaching e. V. in Leipzig, Supervisor, Coach und Lehrsupervisor. 